Originally published as:

Burger, R. "Emerging pathogens and possible threats to blood services",2009," ISBT Science Series", 4, 1, 121 - 123

DOI: 10.1111/j.1751-2824.2009.01231.x

The definitive version is available at http://www3.interscience.wiley.com/journal/122201862/abstract 


\title{
Emerging pathogens and possible threats to blood services
}

\author{
R. Burger \\ Robert Koch Institute, Berlin, Germany
}

\begin{abstract}
New pathogens emerge regularly. These new agents include both bacterial and viral pathogens. Many viral agents have a viremic phase during the course of infection and are transmitted by blood transfusion. New infectious agents provide a major challenge for blood services. The extend and mode of transmission through blood is initially unclear. Serological tests and molecular tools are used to detect infectious donors. A residual risk remains due to the diagnostic window period and sensitivity limits of the tests systems. Some detection systems proved to be extremely helpful, e.g. for HIV, $H C V$ or WNV, but sometimes provide in certain periods of the infection false-negative results due to sensitivity problems or to escape mutants (e.g. Hepatitis B). WNV provides an excellent example, how rapid and efficient blood donation services coped with this new threat for blood safety. Not all emerging infectious agents transmissible by blood represent a threat for the health. A number of putative pathogens turned out later not to be associated with pathogenesis. Prion diseases represent a particular challenge for detection in blood donors. Since the 'normal' cellular form the host protein is widely distributed, the usual nucleic acid amplification systems or serological tests are not feasible. Extremely sensitive tests (picogram range) are required for detection of the pathological protein in the asymptomatic period. Variants of existing viruses e.g. newly emerging Influenza A subtypes with the potential for pandemic spread represent an enormous threat for public health and for the safety of blood. Blood services should take precautions for an influenza pandemic. The mosquito borne Chikungunya virus turned out to be transmitted by blood transfusion and caused many infections in the islands of the Indian Ocean and in India. Newly emerging infectious agents require attention and rapid actions in order to guarantee a safe and reliable blood supply.
\end{abstract}

In the last three decades, on average roughly once a year a new pathogen emerged in one or the other area of the world. A substantial number of these new or re-emerging pathogens continues to remain a threat for the health of the population and may remain with mankind for the foreseeable future. These new agents include both bacterial and viral pathogens like Borrelia, human immunodeficiency virus (HIV), helicobacter, hepatitis C or the agents causing transmissible spongiform encephalopathies. Some of these newly emerging agents were successfully contained and do presently not cause disease, for example, the severe acute respiratory syndrome Corona virus. One may predict that also in the future new pathogens will emerge. Some of these agents were never recognized previously, like severe acute respiratory syndrome or HIV. Various other agents show suddenly a new distribution or a new form of disease manifestation, for example, West Nile virus. A substantial number of pathogens are transmitted from animals to humans, like monkey pox or Nipah virus. In addition, some well known agents cause previously unknown disease, for example, Parvovirus B19, and new forms of previously known agents appear that differ in their properties from previously identified forms, as HIV 1 group O or variant Creutzfeldt-Jakob disease. Finally, one cannot exclude the deliberate release of infectious agents through bioterroristic actions. Many viral agents have a viraemic phase during the course of infection and are therefore transmitted by blood transfusion or cellular blood components and sometimes also plasma products. New infectious agents provide a major challenge for blood services. Initially, there is poor information about the properties of the individual agent and the course of the disease. The extent and mode of transmission through blood is initially unclear and sensitive and specific tests are missing. It requires a substantial amount of time to establish robust tests for screening of blood donations in large numbers. Such tests are required during the asymptomatic period of the disease where seemingly healthy patients still donate blood but are infectious (e.g. West Nile virus). Donor selection criteria are not seldom only partially helpful (e.g. residence in the UK for variant Creutzfeldt-Jakob disease) and inactivation procedures are only useful for a limited number of cellular blood components in contrast to plasma products where effective inactivation procedures exist. The tragic experience with HIV sensitized the population and in many countries a zero risk is - unrealistically - expected. Serological tests and molecular tools are prerequisites to detect infectious donors. A residual risk remains, due to the diagnostic window 
period and sensitivity limits of the tests systems. Some detection systems proved to be extremely helpful, for example, for HIV, HCV or WNV. Sometimes detection systems turn out to be misleading (e.g. identification of the seemingly pathogens SEN, TT virus or hepatitis G) or provide in certain periods of the infection false-negative results due to sensitivity problems or due to escape mutants (e.g. hepatitis B). West Nile virus, a RNA virus and member of the genus Flavivirus serologically related to Japanese encephalitis virus, provides an example for a very rapid and successful spread of a previously existing virus. Recognized 1999, WNV spread within 5 years from the US East coast all the way across the continent to the West coast and is meanwhile endemic. WNV provides an excellent example, how efficient blood donation services coped with this new threat for blood safety. Soon after transient viraemia in asymptomatic infections suggested a transmission of WNV by blood transfusion, the first transmissions through blood were reported in August 2002. Less than a year later, an investigational assay for the screening of blood donors was implemented by detecting WNV RNA in a real-time reverse transcriptase polymerase chain reaction. This assay was applied for the screening of a large number of donations. One year later, virtually all US blood donations were screened by nucleic acid amplification techniques. However, despite rapid establishment of a sensitive and specific assay, breakthrough infections were observed, that is, viraemic donors were false-negative in the screening systems and transmitted WNV despite a low level of viraemia, undetectable in the mini-pool screening. Not all emerging infectious agents transmissible by blood represent a threat for health. A number of putative pathogens isolated from hepatitis patients tested negative in the conventional screening assays (e.g. for Hep A, -B and -C) turned out to be more or less easily transmissible by blood. There were identified by molecular tools from organs or body fluids from patients but turned out later not to be associated with pathogenesis, that is, they are viruses without disease. They are upon improvement of detection systems present sometimes in a substantial percentage of the population (e.g. TT-virus or GBV-C, also named hepatitis G virus). Prion diseases represent a particular challenge for detection in blood donors. For transmissible spongiform encephatopathies (TSE) agents, both serological and molecular tools may not provide a sufficiently reliable screening system and confirmation tests are missing. According to the protein-only hypothesis, these infectious agents represent a host protein in a structurally altered configuration and do not contain nucleic acid. As the 'normal' cellular form of the protein is widely distributed, nucleic acid amplification systems are not applicable. In addition, for detection of a modified host protein, serological tests are extremely difficult to establish. In the asymptomatic period of the disease, only pictogram amounts of the pathological protein are present in the circulation. Correspondingly, extremely sensitive tests are required

that selectively detect only the pathological variant of the protein. Despite these pitfalls, tests may appear on the market in the near future, which achieve specificity through quantitative protease digestion of the cellular protein followed by detection of the pathological variant via enrichment through suitable ligands and subsequently by sensitive indirect detection systems, for example, enzyme-linked immunosorbent assay systems. Protein misfolding cyclic amplification might provide an alternative. In this system an amplification of the pathological protein is achieved. Variants of existing viruses that acquire the potential for pandemic spread provide an enormous threat for public health and for the safety of blood and blood products. Newly emerging influenza A subtypes, for example, H5N1, but also other subtypes may achieve the capacity to spread worldwide. Increased virulence, antigenic shift and the corresponding absent immunity in the population together with the ability to spread from human to human in larger scale provides pandemic capacity. Such a virus could - but must not originate from the presently circulating H5N1 virus. Blood services should take precautions for an influenza pandemic. Presently established emergency plans may not be helpful because of a high number of employees sick for a longer time period or absent because taking care of patients in the family or because of fears to acquire an infection. In the early period of a pandemic, an effective vaccine is probably not available. During an influenza pandemic, the number of donors may drop dramatically due to infectionsor fears of infection. There might be shortage of blood during the pandemic and also exclusion of donors due to antiviral treatment. The time course and extend of viraemia in an influenza infection remains to be determined for the emerging pandemic virus. The duration of the asymptomatic period should be short, that is, only a few days. The period when a blood donation is infectious remains then to be analysed. Experience from other virus subtypes or from the regular seasonal influenza may not apply for the pandemic virus and may not be informative for newly emerging subtypes. The properties of a pandemic virus will only be known when the pandemic does indeed appear. H5N1 virus was found in plasma in a child by reverse transcriptase polymerase chain reaction. The plasma of this severely ill patient was obtained in a terminal period of the disease. During an influenza pandemic, a viraemia in the pre-symptomatic phase of an influenza infection has to be assumed. Due to the short incubation period, the number of influenza transmissions through blood components may remain lower than frequently feared. It is important that blood services develop a concept for maintaining their functions in a pandemic situation. Proper hygiene measures and barrier systems are important and require detailed training of the personnel. 
Prevention of infections may prove to be more efficient than the use of antiviral drugs. In a pandemic situation, shortages of blood donations may frequently be compensated by postponement of elective surgery. Finally, the Chikungunya virus (Alpha virus), transmitted similarly to Dengue virus by mosquitoes, turned out to be transmitted by blood transfusion. This virus caused a substantial number of infections in the islands of the Indian Ocean (La Reunion, Mauritius, Seychelles, Madagascar) and in India. In La Reunion, 20-30\% of the population were infected. Transport of blood from France was necessary to maintain blood supply. Chikungunya cases in the Italian region Emilia Romana caused concern about the spread of Chikungunya to other parts of Europe. Newly emerging infectious agents require particular attention and rapid actions in order to guarantee a safe and reliable blood supply. 